\title{
The CUSUM out-of-control table to monitor changes in fish stock status using many indicators
}

\author{
Pierre Petitgas $^{\mathrm{a}}$ \\ IFREMER, Dép. Ecologie et Modèles pour l'Halieutique (EMH), BP 21105, 44311 Nantes Cedex 3, France
}

Received 23 July 2008; Accepted 16 January 2009

\begin{abstract}
One method to assess fish stocks using a suite of indicators is the traffic light approach. In this approach, the time series of the different indicators are mapped on a common colour scale to highlight alerts that occur when indicators cross reference limit values. Until now, however, the procedure has lacked a statistical framework. Here, we propose the cumulative sum (CUSUM) monitoring scheme as a suitable statistical framework. CUSUM is a statistical process control method that detects deviations from a reference mean, according to defined performance criteria. With the CUSUM monitoring scheme, alarm signals can be triggered when indicators cross defined in-control limits that correspond to defined probabilities of false alarm and non-alarm (i.e., precision and power of the CUSUM monitoring scheme). A table of CUSUM out-of-control deviations is constructed to serve as a diagnostics table. In this table, the deviations in the different indicators are quantitative and given in similar units of variance, which facilitates their integrated assessment. The CUSUM out-of-control table also shows how deviations accumulate over time and thus provides a view of the stock history. The procedure was applied to the North Sea cod stock to illustrate how a fisheryindependent integrated assessment can be achieved using a suite of indicators derived from research survey data. The indicators used were related to the spatial distribution, abundance, length structure, length at maturity and apparent mortality. The stock was found to be outside its reference limits from 2001 and has shown a continued degradation in status since this time.
\end{abstract}

Key words: Indicators / CUSUM / Monitoring / Traffic light approach / Integrated assessment

\section{Introduction}

Fisheries research survey programs provide time series of a wide range of indices relative to different attributes of fish populations. These indices can offer a broad biological basis for indicators to assess fish stock status. In practice, this type of fish stock assessment requires the use of many such indicators (e.g., Link 2005). Moreover, the ecosystem approach to fisheries management considers fish stocks in their ecosystems, which broadens the range of indicators one must consider (e.g., Jennings 2005). The question then arises of how to combine the various results to achieve an integrated assessment. One way of doing this is to use a "traffic light approach" (Caddy 2002, 2004; Caddy et al. 2005; Halliday et al. 2001). In this approach, the different indicators are first mapped on a common colour scale to display whether reference values have been crossed. The use of thresholds and reference points is central to mapping the indicator series into a traffic light table. The assessment is then achieved by some form of integration of the colours, which may use weighting or grouping of the indicator responses. Lastly, management actions are taken based on

\footnotetext{
a Corresponding author: pierre.petitgas@ifremer.fr
}

the assessment and following decision-making rules laid down beforehand. A characteristic of the "traffic light approach" is that it is model-free and presents the time series of fish stock status visually. Caution should be taken when integrating the responses of the indicators to assign the stock to a good or bad status, as many indicators can be correlated. Therefore, simply counting alarms among positively correlated indicators can over-emphasise the seriousness of events. To mitigate the effect of correlation, we used multivariate indicators derived by combining individual indicators (e.g., for spatial distributions), and also considered the response of grouped indicators. This approach allowed different attributes of the stock (e.g., length structure, spatial distribution and abundance) to be monitored using a variety of indicators.

Because survey-derived indicators are noisy, reference points may be crossed simply by chance. A statistical framework is therefore needed to correctly interpret the variability in the indicator time series and detect changes with a defined probability of errors. Prager et al. (2003) suggested estimation of limit values for indicators, based on a probability distribution for the indicators and a defined risk $\alpha$ of finding an infringement when, in fact, none occurred. However, this approach did not consider the risk $\beta$ of not detecting 
that an indicator has exceeded the set threshold. In this paper we suggest the use of the statistical process control scheme CUSUM (e.g., Hawkins and Olwell 1997; Mesnil and Petitgas 2009) as a statistical framework for the traffic light approach. The CUSUM monitoring of an indicator will detect a shift in the mean along the time series with a given precision and power. The CUSUM monitoring scheme explicitly makes a compromise between a low enough probability of false alarm and a powerful enough ability to detect change in the indicator mean value while filtering out inherent variability. In the present study, we monitored a suite of indicators using CUSUM schemes, assembled results in a CUSUM out-ofcontrol table, and then used this table to construct diagnostics on the status of a fish stock. The procedure permitted us to conduct an integrated assessment using a statistical framework.

The original conception of the traffic light approach requires that reference points be defined for each indicator (e.g., Caddy 2004); however, these are not necessarily available or known for a wide range of indicators. Therefore, rather than consider reference values for each indicator, we considered a reference period for the stock, in which this stock was perceived to be in an acceptable state. Such an approach is typical of phase-I in the statistical process control approach (e.g., Montgomery 2005; Mesnil and Petitgas 2009), in which the in-control state is characterised. The consequence for fish stock assessment is that the assessment made is relative to the defined reference period. In-control limits (thresholds) for each indicator are then defined statistically to correspond to the required precision and power of the CUSUM monitoring scheme. In-control limits are therefore not derived from biological reasoning, although the reference period is.

\section{Method}

A reference period is first defined to represent the incontrol (reference) population state. In-control mean and standard deviation for each indicator are estimated for this period. Then, for each indicator, a CUSUM monitoring scheme is tuned to the desired performance to signal deviations from the in-control mean. In this process, the probabilities of falsealarms and non-alarms are converted into the in-control limits that enclose acceptable deviations from the reference mean. Then, each indicator is monitored outside the reference period using the CUSUM scheme. The application of the CUSUM to all indicators produces an array of deviations from the reference mean vector expressed in standard deviation units. This array is the CUSUM diagnostics table, where each column corresponds to the time series of deviations for an indicator. Because the deviations are expressed in units of standard deviation (sd), comparisons between indicators are immediate. Setting all non-alerting deviations to zero, the diagnostics CUSUM table assembles the alerting deviations from the reference means with a + or - sign. In some years, only a small number of indicators may signal alarms, some perhaps with high deviations; in other years, many may signal. As in the traffic light approach, we may either use judgement or an agreed rule to assign the stock in each year to the "incontrol" or "out-of-control" state. This assignment is based on which indicators signal alarm and on how many of them do so. An important characteristic of the CUSUM is that it accumulates deviations over time. The out-of-control deviations in the diagnostics table thus also show historical deviations along the time series. They therefore reveal, for example, how the CUSUM accumulates out-of-control deviations or, on the contrary, how it returns within the in-control limits.

The tuning of the CUSUM monitoring scheme relies on the statistical distribution that is fitted to the indicator. The CUSUM distribution is deduced from the indicator distribution. Then in-control limits are estimated that correspond to the desired statistical performance of the CUSUM scheme (Hawkins and Olwell 1997, chapter 6). When the indicator is Gaussian, the tuning of its CUSUM scheme can follow the procedure described in Mesnil and Petitgas (2009). For a multivariate indicator that is a square Euclidian distance quantifying the departure from a reference, the indicator distribution can be Chi-square. In this case, the tuning of its CUSUM scheme can follow the procedure described in Petitgas and Poulard (2009).

The tuning phase achieves a compromise between the value of the shift in the mean that can be detected by the monitoring scheme and the probability with which it can be detected. Four parameters characterise the performance of the monitoring scheme (e.g., Mesnil and Petitgas 2009):

- The allowance, $k$ (sd units), indicates the amplitude of the fluctuations not retained as significant and that are filtered;

- The decision interval, $h$ (sd units), indicates the in-control limits which, if infringed, will generate an out-of-control signal;

- The (in-control) average run length, IC.ARL or ARL(0), is the average time to a false alarm (units are the time series step);

- The out-of-control average run length, OC.ARL or $\operatorname{ARL}(2 k)$, is the average time before detecting a shift in the mean (units are the time series step).

The average run lengths of the CUSUM scheme can be defined as rates $\left(\right.$ time $^{-1}$ ) and are the inverse of the false-alarm probability and the non-alarm probability of the CUSUM.

\section{Example application}

To illustrate the application in practice, the procedure was applied to the North Sea cod stock for which indices of abundance, length, mortality and spatial distribution have been estimated (Cotter et al. 2009; Woillez et al. 2009) using the survey data of the North Sea International Bottom Trawl Surveys coordinated by the International Council for the Exploration of the Sea (ICES IBTS quarter 1 data). The different steps of the procedure were as follows: estimation of the indicators from the survey data, definition of the reference period and estimation of the in-control mean and variance for each indicator, tuning of the CUSUM scheme for each indicator, application of the CUSUM scheme to each of the indicator time series, assembly of the out-of-control deviations in the CUSUM diagnostics table, and performance of an integrated assessment using a rule to assign the stock to the "in-control" or "out-ofcontrol" state.

The survey time series spanned 21 years, 1985-2005, and fish ages recorded in the survey ranged from 1 to 6 . The estimated indicator series used are available from the EU project 

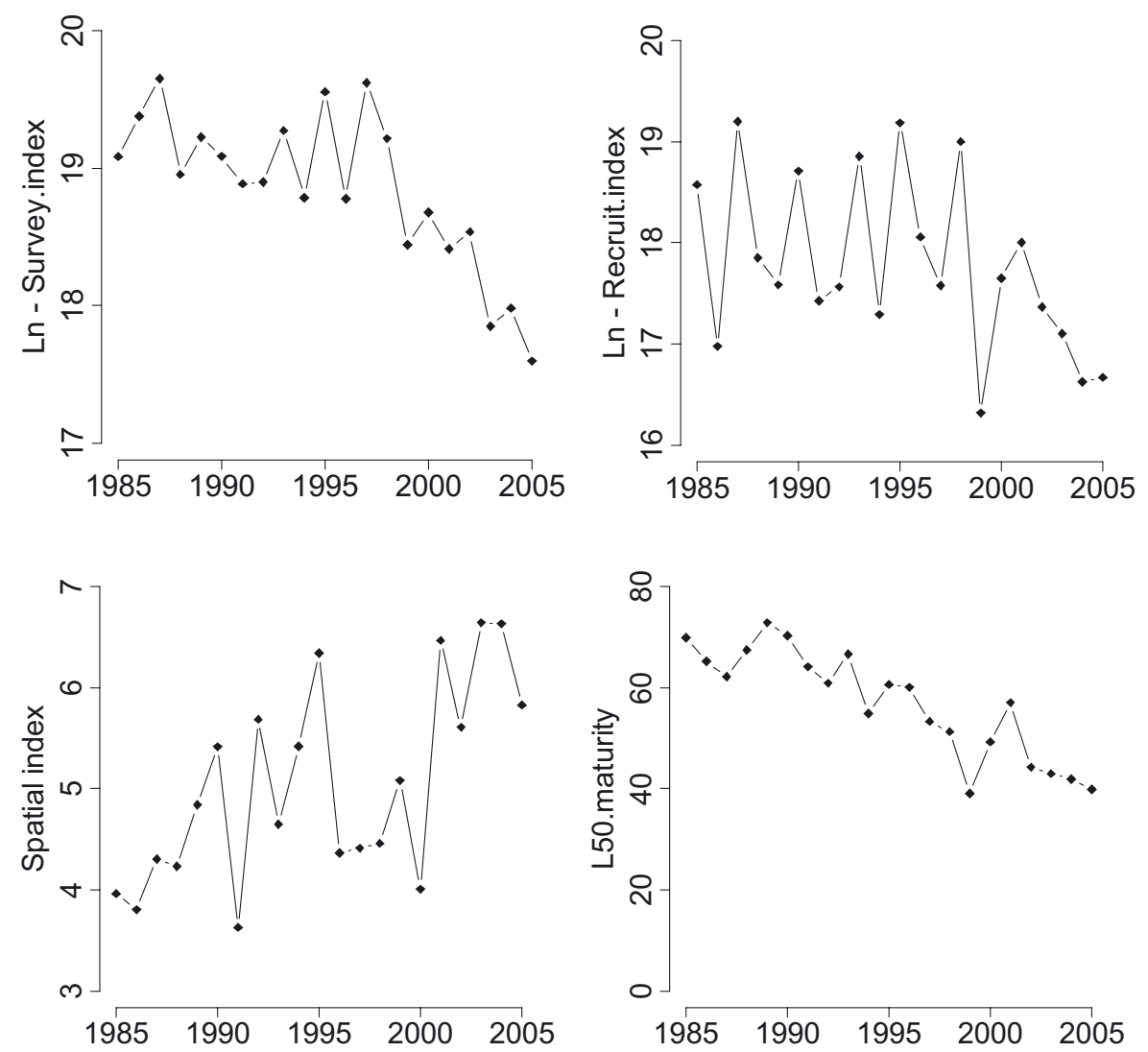

Fig. 1. Indicators of North Sea cod that show a significant shift in their mean along their 1985-2005 time series, supporting the alarm signal indicated by the CUSUM monitoring scheme. Indicators are: Survey index: Log abundance index (numbers of fish); Recruit index: Log abundance at age 2 (numbers of fish); Spatial index: $\sqrt{2 D^{2}}$, where $D^{2}$ is a distance measuring the departure from the average spatial distribution over all ages and is obtained by applying Multi Factor Analysis on the spatial indices at age; L50. maturity: length (cm) at which 50\% of the population is reproductively mature.

FISBOAT website at http://www.ifremer.fr/drvecohal/fisboat/. The indicators of abundance were the survey index (total fish numbers) and the recruit index (numbers at age 2). The indicators of length were the mean (Lbar) and the quartiles (L25, L75) of the fish length distribution. The indicator of maturity was the length at which 50 percent of the fish were sexually mature (L50). The indicator of mortality was the total mortality coefficient $\mathrm{Z}$ estimated by the $\log$ ratio between all individuals aged 1 to 5 in year $t-1$ and those aged 2 to 6 in year $t$. From the eight spatial indicators at age described in Woillez et al. (2009), characterising the spatial distributions at age (longitude and latitude of gravity centre, inertia, anisotropy, patches, positive area, spreading area, equivalent area, microstructure), a multivariate spatial index was estimated using Multi Factor Analysis (Petitgas and Poulard 2009). The spatial index quantified the departure from the mean distribution in the reference period, for each year and over all ages. In all, eight indicator time series were considered to monitor the status of the North Sea cod stock (Figs. 1 and 2): two indicators relative to abundance, three relative to the length structure, one relative to maturity, one for mortality and one multivariate indicator for the spatial distributions at age.

The reference (in-control) period was the first 10 years of the series, 1985-1994, which corresponded to a relatively high abundance in the survey data series, with no particular trend in abundance or recruitment. The reference period was agreed after discussions that took place during an international workshop of the FISBOAT project. The same reference period was applied to all indicators and an in-control mean and standard deviation were estimated for each indicator (Table 1). The distribution of all indicators was considered Gaussian except for the abundance indices, which were assumed to be log-normal, and the multivariate spatial indicator, $D^{2}$, which was considered Chi-square. Abundance indices were log transformed and the spatial indicator was square root transformed $\left(\sqrt{2 D^{2}}\right)$ to approach normality. The CUSUM scheme was then tuned for each indicator (Table 1). Shifts in the mean $(2 k)$ in the order of 2 sd could be reliably detected with good precision (one false alarm every 30 years on average) and power (average detection time lower than 2 years).

The results of applying the CUSUM monitoring scheme to the indicator time series were assembled in an array of out-ofcontrol deviations from the reference mean vector expressed in standard deviation units. This table (Table 2) formed the CUSUM diagnostics table. It indicated that the deviations that could be visually identified on the indicator time series (Figs. 1 and 2) were statistically significant.

Given the variability in the time series of the length and mortality indicators, no change in the mean was detected, except in 1995. In contrast, shifts in the mean were detected for 
Table 1. Parameters of the CUSUM monitoring schemes for North Sea cod spatial and biological indicators. The reference period is 19851994. Parameters are: mean (mu) and standard deviation (sd) in the reference period; allowance (k in sd units); decision interval (h in sd units); average run length (ic.arl, time in years) of the CUSUM to a false alarm (also noted ARL(0)); run length (ic.rl.25, in years) of the CUSUM corresponding to the first quartile of the run length distribution; average run length (oc.arl, in years) of the CUSUM to detect a shift in the mean after it has happened (also noted ARL $(2 k)$ ). Indicators are: Spatial index: $\sqrt{2 D^{2}}$, where $D^{2}$ is a distance measuring the departure from the average spatial distribution over ages 1 to 6 and is obtained by applying multi factor analysis to the spatial indices at age; Survey index: Log abundance index (numbers of fish) for ages 1 to 6; Recruit index: Log abundance at age 2 (numbers of fish); L50 maturity: length (cm) at which $50 \%$ of the population is mature; Lbar: average length $(\mathrm{cm})$ in the population; L25, L75: length values (cm) corresponding to the first and third quartiles of the length distribution; $Z$ : apparent total mortality $\left(\mathrm{y}^{-1}\right)$ over ages 1 to 5 .

\begin{tabular}{|c|c|c|c|c|c|c|c|c|c|}
\hline \multirow[t]{2}{*}{ Parameters } & & \multicolumn{8}{|c|}{ Indicators } \\
\hline & & $\begin{array}{l}\text { Spatial } \\
\text { Index }\end{array}$ & $\begin{array}{l}\text { Survey } \\
\text { Index }\end{array}$ & $\begin{array}{l}\text { Recruit } \\
\text { Index }\end{array}$ & $\begin{array}{c}\text { L50 } \\
\text { Maturity }\end{array}$ & Lbar & $\mathrm{L} 25$ & $\mathrm{~L} 75$ & $\bar{Z}$ \\
\hline Mean & $\mathrm{mu}$ & 4.60 & 19.12 & 18.00 & 65.44 & 34.77 & 20.69 & 41.70 & 1.12 \\
\hline Standard deviation & $\mathrm{sd}$ & 0.73 & 0.26 & 0.77 & 5.24 & 4.80 & 5.16 & 6.45 & 0.44 \\
\hline Allowance & $\mathrm{k}$ & 1 & 1.3 & 0.9 & 1.1 & 1.2 & 0.9 & 0.8 & 1.0 \\
\hline Decision interval & $\mathrm{h}$ & 1.2 & 1.0 & 1.0 & 1.1 & 1.0 & 1.0 & 1.2 & 1.0 \\
\hline In-control average run length (1qt) & ic.arl & 51.8 & 79.3 & 27.5 & 56.2 & 60.0 & 27.5 & 30.0 & 35.3 \\
\hline In-control run length & ic.rl.25 & 16 & 23.0 & 8.0 & 16.0 & 17.0 & 8.0 & 9.0 & 10.0 \\
\hline Out-of-control average run length & oc.arl & 2.0 & 1.5 & 1.9 & 1.8 & 1.6 & 1.9 & 2.3 & 1.8 \\
\hline
\end{tabular}

Table 2. CUSUM diagnostics table for North Sea cod using spatial and biological population indicators. Values are the out-of-control deviations from the reference mean for each indicator in standard deviation units. The reference period is 1985-1994. The procedure signals an alarm from 2001 onwards. Indicators are: Spatial index: $\sqrt{2 D^{2}}$, where $D^{2}$ is a distance measuring the departure from the average spatial distribution over ages 1 to 6 and is obtained by applying Multi Factor Analysis on the spatial indices at age; Survey index: Log abundance index (numbers of fish) for ages 1 to 6; Recruit index: Log abundance at age 2 (numbers of fish); L50 maturity: length (cm) at which 50\% of the population is mature; Lbar: average length $(\mathrm{cm})$ in the population; L25, L75: length values $(\mathrm{cm})$ corresponding to the first and third quartiles of the length distribution; $Z$ : apparent total mortality $\left(\mathrm{y}^{-1}\right)$ over ages 1 to 5 .

\begin{tabular}{|c|c|c|c|c|c|c|c|c|c|}
\hline Year & $\begin{array}{l}\text { Spatial } \\
\text { Index }\end{array}$ & $\begin{array}{l}\text { Survey } \\
\text { Index }\end{array}$ & $\begin{array}{l}\text { Recruit } \\
\text { Index }\end{array}$ & $\begin{array}{c}\text { L50 } \\
\text { Maturity }\end{array}$ & Lbar & L25 & L75 & $Z$ & Diagnostics \\
\hline 1985 & 0.00 & 0.00 & 0.00 & 0.00 & 0.00 & 0.00 & 0.00 & 0.00 & Ref \\
\hline 1986 & 0.00 & 0.00 & 0.00 & 0.00 & 0.00 & 0.00 & 0.00 & 0.00 & Ref \\
\hline 1987 & 0.00 & 0.00 & 0.00 & 0.00 & 0.00 & 0.00 & 0.00 & 0.00 & Ref \\
\hline 1988 & 0.00 & 0.00 & 0.00 & 0.00 & 0.00 & 0.00 & 0.00 & 0.00 & Ref \\
\hline 1989 & 0.00 & 0.00 & 0.00 & 0.00 & 0.00 & 0.00 & 0.00 & 0.00 & Ref \\
\hline 1990 & 0.00 & 0.00 & 0.00 & 0.00 & 0.00 & 0.00 & 0.00 & 0.00 & Ref \\
\hline 1991 & 0.00 & 0.00 & 0.00 & 0.00 & 0.00 & 0.00 & 0.00 & 0.00 & Ref \\
\hline 1992 & 0.00 & 0.00 & 0.00 & 0.00 & 0.00 & 0.00 & 0.00 & 0.00 & Ref \\
\hline 1993 & 0.00 & 0.00 & 0.00 & 0.00 & 0.00 & 0.00 & 0.00 & 0.00 & Ref \\
\hline 1994 & 0.00 & 0.00 & 0.00 & 0.00 & 0.00 & 0.00 & 0.00 & 0.00 & Ref \\
\hline 1995 & 1.53 & 0.00 & 0.00 & 0.00 & 0.00 & 0.00 & 0.00 & 0.00 & \\
\hline 1996 & 0.00 & 0.00 & 0.00 & 0.00 & 0.00 & 0.00 & 0.00 & 0.00 & \\
\hline 1997 & 0.00 & 0.00 & 0.00 & -1.85 & -1.84 & -1.03 & -2.21 & 0.00 & \\
\hline 1998 & 0.00 & 0.00 & 0.00 & -3.44 & 0.00 & 0.00 & -2.00 & 0.00 & \\
\hline 1999 & 0.00 & -1.27 & -1.30 & -7.36 & 0.00 & 0.00 & 0.00 & 0.00 & \\
\hline 2000 & 0.00 & -1.65 & 0.00 & -9.34 & 0.00 & 0.00 & 0.00 & 0.00 & \\
\hline 2001 & 1.57 & -3.04 & 0.00 & -9.84 & 0.00 & 0.00 & 0.00 & 0.00 & Alarm \\
\hline 2002 & 1.96 & -3.96 & 0.00 & -12.78 & 0.00 & 0.00 & 0.00 & 0.00 & Alarm \\
\hline 2003 & 3.77 & -7.48 & 0.00 & -15.95 & 0.00 & 0.00 & 0.00 & 0.00 & Alarm \\
\hline 2004 & 5.56 & -10.50 & -1.18 & -19.33 & 0.00 & 0.00 & 0.00 & 0.00 & Alarm \\
\hline 2005 & 6.26 & -14.97 & -2.02 & -23.10 & 0.00 & 0.00 & 1.23 & & Alarm \\
\hline
\end{tabular}

L50 at maturity, the survey index of abundance and the spatial index. The deviations were negative for L50 at maturity and the abundance index meaning that, in the North Sea cod population, the mature fish got smaller and smaller with time and abundance decreased. The spatial index (being a distance) was only positive and therefore its deviation was also only positive: the North Sea cod population showed a spatial distribution over all ages that became increasingly different from that of the reference years. The deviations accumulated with time, reaching more than $20 \mathrm{sd}$ units for L50 at maturity, more than 10 sd units for the abundance and more than 6 sd units for the spatial index. Clearly, the stock accumulated large deviations from its reference state. For the CUSUM to return to within in-control limits, opposite deviations would need to accumulate. This could be achieved by large deviations in a small time period or smaller ones over a longer time period. 

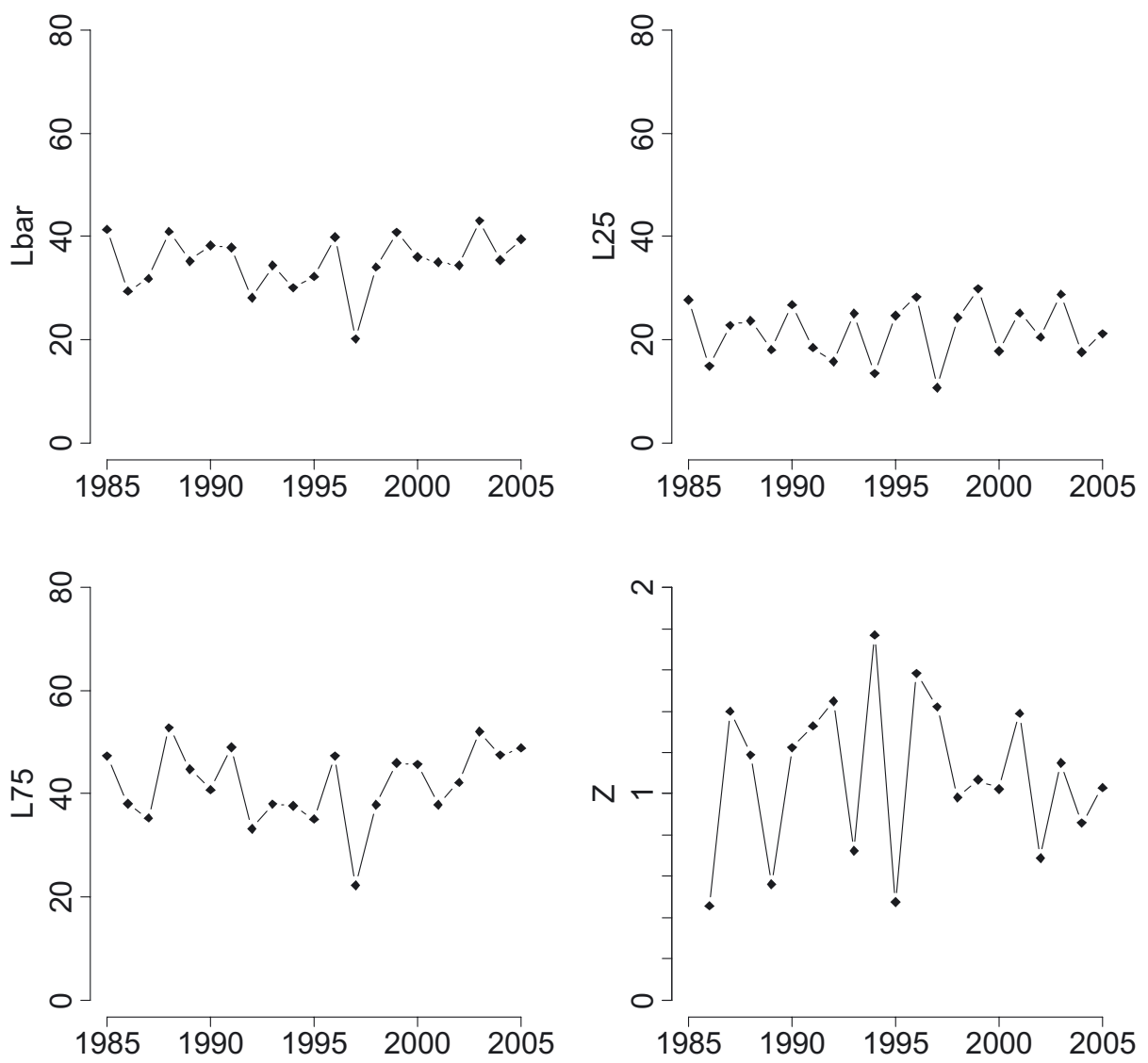

Fig. 2. Indicators of North Sea cod that show no shift in their means through the 1985-2005 time series. Indicators are: Lbar: mean length (cm) in the population; L25, L75: length values $(\mathrm{cm})$ at the first and third quartiles of the length distribution; $Z$ : total apparent mortality $\left(\mathrm{y}^{-1}\right)$ over ages 1 to 5 .

As characterised in the CUSUM diagnostic table (Table 2), the state of North Sea cod continuously degraded from 2001 onwards, with no sign of a return to the reference state.

To assign the stock in each year to the "in-control" or "outof-control" state (Table 2, right column), the following rule was used. The indicators were grouped into 5 categories (stock attributes): abundance, spatial distribution, maturity, length and mortality. The stock was considered as "out-of-control" for any one year if the CUSUM scheme signalled for indicators belonging to at least 3 categories. Following this rule, the stock was repeatedly out of control from 2001 and the deviations became continuously worse. Just before 2001, in the period 1997-2001, there were already signs of the stock being close to out-of-control. It seems that there was a sequence of deterioration as the indicators signalled progressively: (1) from 1997 length at maturity constantly decreased, (2) from 1999 abundance constantly decreased, and (3) from 2001 the spatial distribution signalled increasing departure from the reference spatial pattern (Table 2, Fig. 3). The state of the stock indicated by the CUSUM diagnostics table was consistent with assessments carried out by ICES (e.g. 2003) based on catch-at-age analyses, which showed that the spawning stock had consistently been below the limit reference point since 1999. In 2001, ICES advised a closure of the directed fishery. The present procedures broaden the biological basis of that assessment.

\section{Discussion - Conclusion}

The CUSUM monitoring scheme provides a statistical framework for the traffic light approach. It allows monitoring of changes in indicator time series. The method explicitly shows the significant shift in the mean that can be detected, together with the statistical significance with which it can be detected. The performance statistics of the monitoring scheme are based on the statistical distribution that the indicator is assumed to follow. The CUSUM out-of-control diagnostics table assembles the out-of-control deviations for all indicators. The deviations are quantitative and given in similar units for all indicators, allowing comparisons between indicators. Evolution over time can also be characterised as the CUSUM accumulates deviations. On these bases, the CUSUM diagnostics table permits an integrated assessment with a historical perspective. The application to North Sea cod illustrates how survey-based indicators relating to spatial distribution, abundance, length structure, maturity and apparent mortality could be integrated into one single assessment. Across all indicators, the false alarm rate was greater than 30 years and the nonalarm rate was lower than 2 years. The deviations in the mean that could be detected with such performance were close to 2 standard deviations. The result of the assessment was that the 


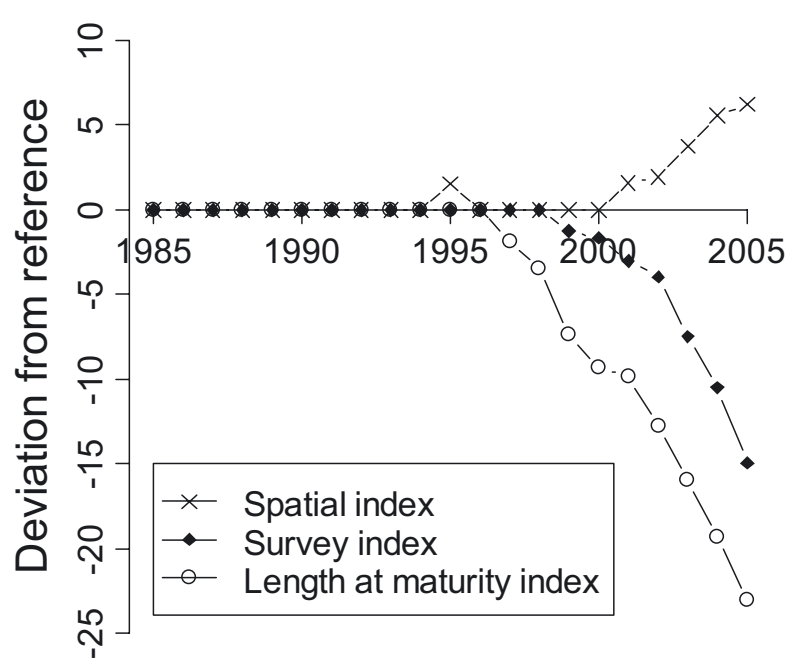

Fig. 3. Evolution of the North Sea cod stock as characterised by the CUSUM monitoring scheme 1985-2005. Deviations are relative to the reference mean and expressed in units of standard deviation. Reference means and standard deviations are estimated for the reference period 1985-1994. The statistical performance of the monitoring scheme is presented in Table 1.

stock was outside its reference limits from 2001 onwards, with continuous degradation of its status.

The CUSUM diagnostics table is designed to monitor changes in the mean. This is a crucial and neutral component of the assessment (Spellerberg 2005) which also serves to assess the impact of past management actions. The indicators used here related to different attributes of a fish stock, but the method is applicable to a larger set of indicators relating to an entire ecosystem. It could serve for fish stock monitoring with an ecosystem perspective (e.g., Steele 1996) as well as for integrated ecosystem assessment (e.g., Link et al. 2002).

Acknowledgements. This study was carried out with financial support from the European Union, project FISBOAT, contract FP6 No.502572. We are grateful to ICES for the maintenance of and access to the IBTS database. The spatial indicators were calculated for North Sea cod by C. Deerenberg (IMARES) within the FISBOAT project, using the $\mathrm{R}$ language codes developed at Centre de Géostatistique and IFREMER. Indicator time series and codes are available on the FISBOAT website at http://www.ifremer.fr/drvecohal/fisboat/.

\section{References}

Caddy J.F., 2002, Limit reference points, traffic lights, and holistic approaches to fisheries management with minimal stock assessment input. Fish. Res. 56, 133-137.

Caddy J.F., 2004, Current usage of fisheries indicators and reference points, and their potential application to management of fisheries for marine invertebrates. Can. J. Fish. Aquat. Sci. 61, 1307-1324.

Caddy J.F., Wade E., Surette T., Hebert M., Moriyasu M., 2005, Using an empirical traffic light procedure for monitoring and forecasting in the Gulf of St. Lawrence fishery for the snow crab, Chionoecetes opilio. Fish. Res. 76, 123-145.

Cotter J., Mesnil B., Witthames P., Uriarte A., Parker-Humphreys M., 2009 , Notes on nine biological indicators estimable from trawl surveys with an illustrative assessment for North Sea cod. Aquat. Living Resour. 22, 135-153.

Halliday R.G., Fanning L.P., Mohn R.K., 2001, Use of the traffic light method in fishery management planning. Canadian Science Advisory Secretariat Res. Doc. 2001/108, pp. 1-41.

Hawkins D.M., Olwell D.H., 1997, Cumulative sum charts and charting for quality improvement. Springer Verlag, New York.

ICES 2003, Report of the ICES Advisory Committee on Fishery Management, 2003. ICES Coop. Res. Rep. 261, 3.

Link J.S., 2005, Translating ecosystem indicators into decision criteria. ICES J. Mar. Sci. 62, 569-576.

Link J.S., Brodziak J.K.T., Edwards S.F., Overholtz W.J., Mountain D., Jossi J.W., Smith T.D., Fogarty M.J., 2002, Marine ecosystem assessment in a fisheries management context. Can. J. Fish. Aquat. Sci. 59, 1429-1440.

Mesnil B., Petitgas P., 2009, Detection of changes in time-series of indicators using CUSUM control charts. Aquat. Living Resour. 22, 187-192.

Montgomery D.C., 2005, Introduction to statistical quality control. $5^{\text {th }}$ edn. Wiley, New York.

Petitgas P., Poulard J.-C., 2009, A multivariate indicator to monitor changes in the spatial patterns of age-structured fish populations. Aquat. Living Resour. 22, 165-171.

Prager M.H., Porch C.E., Shertzer K.W., Caddy J.S., 2003, Targets and limits for management of fisheries: a simple probabilitybased approach. N. Am. J. Fish. Manage. 23, 349-361.

Spellerberg I., 2005, Monitoring ecological change. $2^{\text {nd }}$ edn. Cambridge University Press, Cambridge.

Steele J.H., 1996, Regime shifts in fisheries management. Fish. Res. $25,19-23$.

Woillez M., Rivoirard J., Petitgas P., 2009, Notes on survey-based spatial indicators for monitoring fish populations. Aquat. Living Resour. 22, 155-164. 